\title{
Improving Breast and Colon Cancer Screening Rates: A Comparison of Letters, Automated Phone Calls, or Both
}

\author{
Lindsay Phillips, MD, Samantha Hendren, MD, MPH, Sharon Humiston, MD, MPH, \\ Paul Winters, MS, and Kevin Fiscella, MD, MPH
}

Purpose: Low-cost interventions to improve cancer screening among primary care patients are needed. The comparative effectiveness of personalized letters, automated telephone calls, and both on breast cancer (BC) and colorectal cancer (CRC) screening is not known.

Methods: A pragmatic, randomized, controlled trial was conducted in 2011 to 2012 . Eligible primary care patients were women ages 50 to 74 years who were past due for mammography and men or women who were past due for mammography or CRC screening of any kind (>12 months since last fecal occult blood test, $>5$ years since last sigmoidoscopy/double-contrast barium enema, or $>10$ years since last colonoscopy), respectively. Participants were randomized to 1 of 3 interventions: personalized mailed letters, automated telephone calls, or both. The primary outcome was medical record documentation of a completed mammogram or CRC screening within 36 weeks of randomization. We estimated the costs of each intervention and calculated the marginal cost-effectiveness per person screened.

Results: The crude screening rates for BC were $19 \%, 22 \%$, and $37 \%$ and for CRC were $17 \%, 14 \%$, and $24 \%$ for the letter, automated call, and combined (letter/automated call) groups, respectively. The combined intervention group had a statistically higher screening rate $(P<.05)$ compared with either of the single intervention groups (letter only or automated call) for both BC and CRC in both the crude and adjusted analyses. The combined intervention costs $\$ 5.11$ per additional person screened for $\mathrm{BC}$ and \$13.14 per additional person screened for CRC.

Conclusion: In a primary care practice, letters plus automated telephone calls are better than either alone in increasing cancer screening rates among patients who are overdue for screening. These findings suggest the promise of a relatively inexpensive intervention to improve cancer screening. $(\mathrm{J}$ Am Board Fam Med 2015;28:46-54.)

Keywords: Cancer Screening, Colorectal Cancer, Mammography

Rates of screening for breast cancer (BC) and colorectal cancer (CRC) remain suboptimal, particularly among poor and minority patients. ${ }^{1-3} \mathrm{BC}$

This article was externally peer reviewed.

Submitted 13 June 2014; revised 22 August 2014; accepted 11 September 2014.

From the Department of Family Medicine, University of Rochester Medical Center, Rochester, NY (LP, PW, KF); the Department of Surgery, University of Michigan, Ann Arbor (S. Hendren); and the Department of Pediatrics, Children's Mercy Hospitals and Clinics, Kansas City, MO (S. Humiston).

Funding: This project was supported by the American Cancer Society (RSGT-08-077-01-CPHPS) and the Agency for Healthcare Research and Quality (1 K18 HS022440-01). Conflict of interest: none declared.

Corresponding author: Kevin Fiscella, MD, MPH, 1381 South Ave, Rochester, NY 14620 (E-mail: Kevin_Fiscella@urmc. rochester.edu). screening, based on self-report of a mammogram within 2 years, was $72 \%$ in 2010, whereas selfreported rates of CRC screening were only $64 \% .^{1,2}$ Performance measures for both are now included in Healthcare Effectiveness Data and Information Set quality measures for primary care. ${ }^{4}$ Beyond strong recommendations by clinicians to patients, ${ }^{5}$ however, which strategy or combination of strategies is most effective for improving rates is not clear.

Most cancer screening promotion studies have been conducted among patients who are recruited into and consented to participate in studies. The generalizability of the findings to real-world patient populations is uncertain, however, because the recruitment process introduces a significant consent 
bias $^{6,7}$ by excluding patients who are harder to reach because of an incorrect address or phone number, infrequent office visits, or attitudinal factors. These may be many of the same patients who are at risk for omission of screening. To address this concern, several recent studies obtaining waivers for informed consent (pragmatic randomized trials) have reported the effects of interventions to promote cancer screening. ${ }^{8-14}$ Together, these findings suggest that personal letters from physicians, ${ }^{8,15}$ mailed fecal occult blood testing (FOBT) kits, ${ }^{10,11,16}$ and phone counseling (often following a letter), 9,12,13,15 improve BC and CRC screening rates.

High-cost, personnel-intense interventions (eg, live phone calls to patients) may not be affordable in many primary practices. Lower-cost interventions such as automated phone calls and letters may be more feasible. We identified only 2 randomized trials of standard automated phone calls. ${ }^{14,17}$ One showed improvement in CRC screening rates ${ }^{14}$ and the other showed improvement in BC screening rates. ${ }^{17}$ Interestingly, a study using voice recognition to tailor the recommended CRC screening modality showed no effect. ${ }^{18}$ A systematic review confirmed the benefit of mailed reminders for BC and CRC screening. ${ }^{19}$ Our own research suggests that multimodal interventions are effective, ${ }^{20,21}$ but which component(s) of the interventions really prompted increases in screening rates is unclear from prior studies.

The goal of this study was to understand the differential effects of low-cost automated telephone and mailed interventions on cancer screening rates in a primary care practice. A pragmatic randomized trial design was used to mimic real-world clinical circumstances. We specifically compared the effectiveness and costs of mailed personalized letters, personalized automated telephone calls, or both on rates of cancer screening among primary care patients "past due" for mammography or CRC screening. We hypothesized that the combined intervention would be more effective than either alone. Samples of letters to participants are available upon request from the corresponding author.

\section{Methods}

\section{Practice Setting}

We chose a mixed urban-suburban family medicine practice in which to conduct this trial. The hospital-owned, nonacademic practice consisted of 6 physicians (3.4 full-time equivalents) caring for about 5000 patients. At the time of the study (2011 to 2012) the US Preventive Services Task Force had just updated mammogram guidelines to recommend starting screening at age 50 years, ${ }^{22}$ so outreach focused on women 50 to 74 years old. Colorectal screening assessment was done for all men and women 50 to 74 years old. Before the intervention, cancer screening promotion consisted of provider referral and recommendation in the context of a visit. Baseline screening rates in the practice were $67 \%$ for BC screening and $54 \%$ for CRC screening. While these are lower than national self-reported rates, ${ }^{1,2}$ self-reports tend to overestimate rates of screening compared with claims data. ${ }^{23,24}$

\section{Participants}

We identified potentially eligible patients using a 2-step process. First, the practice queried their electronic practice management system based on age, sex, and last visit to the practice. Next, data were manually reviewed to ascertain the date and type(s) of the last cancer screening(s). Participant eligibility criteria for enrollment in the study included (1) being a registered patient at the study clinic; (2) being an active patient at the practice (having at least 1 visit to the practice in the past 2 years); (3) being 50 to 74 years old; and (5) being past due for mammography or CRC screening based on medical record documentation. We defined "past due for mammography" as $>30$ months from the last mammogram. We defined "past due for CRC screening" as $>10$ years from the last colonoscopy (unless recommended earlier by the consultant performing the procedure) or, in the absence of colonoscopy results, $>12$ months from past guaiac FOBT or fecal immunochemical test (FIT) or $>5$ years from the last double-contrast barium enema or flexible sigmoidoscopy. Women could be enrolled based on either (or both) BC or CRC screening criteria. All patients meeting the criteria were included in the study; the institutional review board waived informed consent (see the Informed Consent section below) to allow results to be more generalizable to practice.

Patients were excluded from the study if they were deemed to be at higher risk for cancer by physician experts on the team or were uninsured. Our previous research showed that interventions caused smaller absolute increases in screening among uninsured pa- 
tients. ${ }^{20,21}$ The conditions indicating a higher risk of cancer based on chart data abstraction included prior cancer, premalignant conditions (eg, ulcerative colitis, familial polyposis), inadequately evaluated breast masses, positive FOBT/FIT result at last testing, or a first-degree relative with a previous diagnosis of $\mathrm{BC}$ or CRC. Higher-risk patients and those without insurance who were past due were not randomized but were given the most intensive intervention, that is, both a letter and an automated phone call, and were not analyzed.

\section{Intervention}

Figure 1 describes the participant flow and timeline for the 3 intervention groups. All interventions were delivered solely in English.

\section{Group 1}

Participants were sent a personalized letter, signed by the patient's physician, explaining that (1) the patient was past due for cancer screening; (2) the importance of cancer screening; (3) how to schedule the screening; (4) the name and telephone number of the outreach worker available to assist patients with arranging screening; and (5) the availability of free mammography and CRC screening (FIT) for the uninsured/underinsured through a state-sponsored program, which was included to address underinsurance, and for patients who might have become uninsured following randomization. This content was based on our preliminary work and input from the patient community advi-

\section{Figure 1. Study flow diagram.}

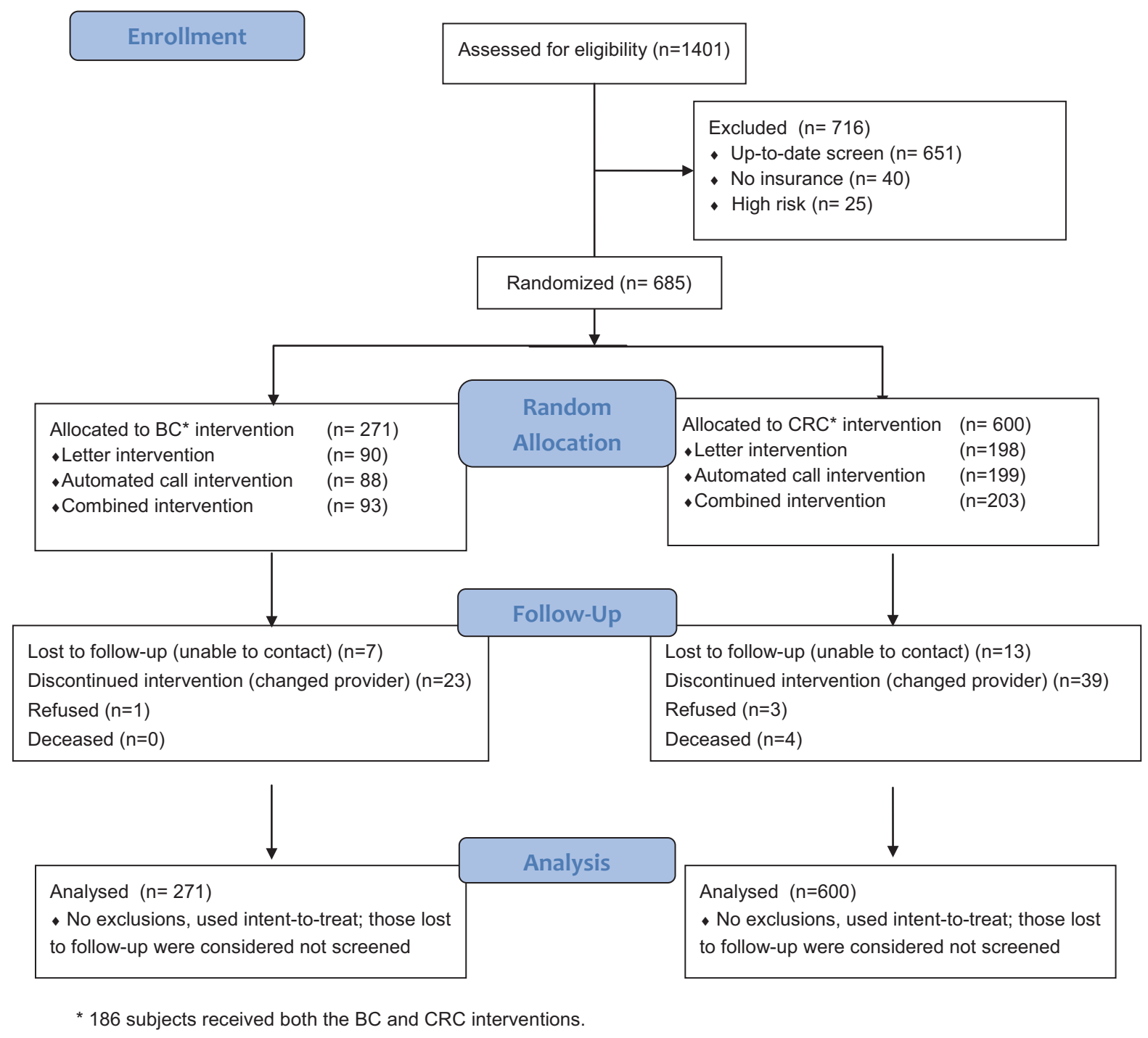


sory board. If still unscreened at 10 weeks, participants received a second letter and FIT kit with a stamped and preaddressed envelope.

\section{Group 2}

Participants received automated telephone calls in up to 3 waves through a commercial vendor. Automated telephone calls were attempted at varying times (up to 5 times) until a person or an answering machine responded during the first wave (week 1). These calls were repeated during the second wave (week 5). Patients who remained unscreened following a reassessment of screening (week 10) received a third wave (weeks 12 to 14). The automated messages contained content similar to that in the letter, including a number to call if they wanted a FIT kit to be mailed.

\section{Group 3}

Participants received both of the interventions described above. Women eligible for both interventions received 1 letter indicating they were past due for both screenings and/or 2 separate automated calls indicating they were past due for mammography and CRC screening (to avoid longer, complex automated calls).

\section{Informed Consent}

The protocol was approved the University of Rochester Institutional Review Board, which waived informed consent by patients. This waiver, similar to those obtained in previous trials of pragmatic cancer screening reminders, is based on the use of standard quality improvement interventions that involve minimal risk to participants and the inability to conduct a pragmatic trial of these interventions in the absence of such a waiver. The study is registered at www. clinicaltrials.gov (identifier NCT00818857).

\section{Measures \\ Covariates}

Trained research assistants abstracted the following data from the electronic health record: patient's age, sex, race/ethnicity, payment/insurance type, number of current medications (as a proxy for comorbidity), and ZIP code of residence, which was converted to median household income based on data from the 2000 US Census.

\section{Primary Outcome}

The primary outcome was medical record documentation (based on manual abstraction of electronic health records) of completed mammography or CRC screening 36 weeks after randomization (approximately 3 months after the end of interventions). Research assistants, who were blinded to the intervention, abstracted data (screening date and results available by week 36). Patients were considered "screened" for BC based on the presence of a mammography report or for CRC based on the presence of a FOBT, FIT, colonoscopy, flexible sigmoidoscopy, or double-contrast barium enema report.

\section{Costs}

We considered all costs involved in implementing each of these interventions, including both the costs of the materials (Article, printing, postage, vendor cost for automated telephone calls) and personnel time involved in preparing lists, letters, and mailing kits. We did not include the study costs of data abstraction.

\section{Randomization and Blinding}

After confirming eligibility through medical record abstraction, each participant was assigned a unique study identification number. A statistician, who was offsite and blinded to the patients' identities, assigned participants equally to 1 of the 3 intervention groups using a computer-generated random number algorithm. Randomization was stratified by the type of screening(s) for which the participants were past due (BC, CRC, or both). Patients who were eligible for mammography and CRC interventions were randomized once and received interventions targeting both mammography and CRC screening. The office clinicians and study staff were blinded to group assignment.

\section{Statistical Analysis}

We compared baseline characteristics of patients in each the 3 intervention groups using the $\chi^{2}$ test for binary variables and $t$ tests for continuous variables. We defined statistical significance as $P<.05$. All participants were analyzed in the originally assigned study group based on intention to treat. We compared postintervention screening rates between the 3 randomized groups using the Pearson $\chi^{2}$ test. We calculated both unadjusted (crude) and adjusted rates. In the adjusted analyses we adjusted for 
small differences in age, sex, race/ethnicity, insurance status, and household income between groups. All statistical analyses were performed using SAS software version 9.3 (SAS Institute Inc., Cary, NC).

\section{Results}

\section{Baseline Characteristics}

The characteristics of the mammography screening participants are shown in Table 1, and characteristics of the CRC screening participants are shown in Table 2. All BC screening and $62 \%$ of CRC screening subjects were women. The mean age of all subjects was 58 years. Most participants were nonHispanic white $(78 \%)$; fewer were black $(13 \%)$ or other (9\%). Most patients had private insurance (63\%), followed by Medicare (25\%) and Medicaid (12\%). Approximately $20 \%$ resided in ZIP codes with a median annual household income $<\$ 30,000$. By definition, rates for both mammography and CRC screening were $0 \%$ before the intervention in each randomization group.

\section{Primary Outcome Measures}

Results are shown in Table 3. The crude screening rates for BC were 19\%, 22\%, and 37\% and for CRC were $17 \%, 14 \%$, and $24 \%$ for the letter, automated call, and combined (letter and automated call) groups, respectively. The adjusted screening rates for $\mathrm{BC}$ were $20 \%, 24 \%$, and $39 \%$ and for CRC were $20 \%$, $16 \%$, and $28 \%$ for the letter, automated call, and combined (letter and automated call) groups, respectively. Overall, $7 \%$ of FIT kits mailed were completed and processed, contributing to one third of all CRC screenings. The combined intervention group had a statistically higher screening rate $(P<.05)$ compared with either of the single intervention groups (automated call only or letter only) for both BC and CRC. Results remained significant for multiple comparisons using the Hochberg and false discovery rate methods. There was no statistical difference between the 2 single interventions. We observed no significant differences in effects across patient race and insurance, though our power was limited. Women past due for both screenings showed significantly lower rates $(P<$ $.05)$ of screening following any intervention than women past due for only 1 screening, possibly reflecting reluctance to undergo cancer screening.

\section{Cost and Cost-Effectiveness}

For group 1, the cost of mammography mailings was $\$ 2.36$ per patient per mailing. The total cost of mailed CRC letters and FIT kits, including prepa-

Table 1. Baseline Characteristics of Patients Past Due for Mammography Screening By Intervention Group

\begin{tabular}{|c|c|c|c|c|}
\hline \multirow[b]{2}{*}{ Characteristics } & \multicolumn{3}{|c|}{ Intervention } & \multirow[b]{2}{*}{$\begin{array}{c}P \\
\text { Value }\end{array}$} \\
\hline & $\begin{array}{l}\text { Group } 1 \text { (Letter) } \\
\quad(\mathrm{n}=90)\end{array}$ & $\begin{array}{l}\text { Group } 2 \text { (Automated Phone } \\
\text { Call) }(\mathrm{n}=88)\end{array}$ & $\begin{array}{c}\text { Group } 3 \text { (Letter and Automated } \\
\text { Phone Call })(\mathrm{n}=93)\end{array}$ & \\
\hline Age (years) & & & & .5095 \\
\hline $50-59$ & $57.8(52)$ & $59.1(52)$ & $65.6(61)$ & \\
\hline$\geq 60$ & $42.2(38)$ & $40.9(36)$ & $34.4(32)$ & \\
\hline Race/ethnicity & & & & .9718 \\
\hline Non-Hispanic white & $77.9(67)$ & $79.8(67)$ & $76.3(71)$ & \\
\hline Non-Hispanic black & $11.6(10)$ & $9.5(8)$ & $12.9(12)$ & \\
\hline Other (eg, Hispanic) & $10.5(9)$ & $10.7(9)$ & $10.8(10)$ & \\
\hline Household income & & & & .8252 \\
\hline$\geq \$ 40,000$ & $48.9(44)$ & $52.3(46)$ & $52.7(49)$ & \\
\hline$\$ 30,000-\$ 39,999$ & $26.7(24)$ & $28.4(25)$ & $22.6(21)$ & \\
\hline$<\$ 30,000$ & $24.4(22)$ & $19.3(17)$ & $24.7(23)$ & \\
\hline Insurance* & & & & .0034 \\
\hline Private & $48.9(44)$ & $51.1(45)$ & $69.9(65)$ & \\
\hline Medicare & $38.9(35)$ & $26.1(23)$ & $19.4(18)$ & \\
\hline Medicaid & $12.2(11)$ & $22.7(20)$ & $10.7(10)$ & \\
\hline Preintervention screening rates* & $0(0)$ & $0(0)$ & $0(0)$ & NA \\
\hline
\end{tabular}

Data are \% (n). Small discrepancies between the total number of patients in a group and the summed numbers in the columns reflect data missing for that variable.

*By design, patients without insurance or who were up to date on breast cancer and colorectal cancer screening were excluded from the study. NA, not applicable. 
Table 2. Baseline Characteristics of Patients Past Due for Colorectal Cancer Screening By Intervention Group

\begin{tabular}{|c|c|c|c|c|}
\hline \multirow[b]{2}{*}{ Characteristics } & \multicolumn{3}{|c|}{ Intervention } & \multirow[b]{2}{*}{$\begin{array}{c}P \\
\text { Value }\end{array}$} \\
\hline & $\begin{array}{l}\text { Group } 1 \text { (Letter) } \\
\quad(\mathrm{n}=198)\end{array}$ & $\begin{array}{l}\text { Group } 2 \text { (Automated Phone } \\
\text { Call) }(\mathrm{n}=199)\end{array}$ & $\begin{array}{c}\text { Group } 3 \text { (Letter and Automated } \\
\text { Phone Call) }(\mathrm{n}=203)\end{array}$ & \\
\hline Sex & & & & .7824 \\
\hline Female & $62.63(124)$ & $63.3(126)$ & $60.1(122)$ & \\
\hline Male & $37.4(74)$ & $36.7(73)$ & $39.9(81)$ & \\
\hline Age (years) & & & & .2677 \\
\hline $50-59$ & $64.1(127)$ & $68.3(136)$ & $60.6(123)$ & \\
\hline$\geq 60$ & $35.9(71)$ & $31.7(63)$ & $39.4(80)$ & \\
\hline Race/ethnicity & & & & .7464 \\
\hline Non-Hispanic black & $14.6(28)$ & $13.0(25)$ & $12.4(25)$ & \\
\hline Other (eg, Hispanic) & $7.3(14)$ & $10.4(20)$ & $7.5(15)$ & \\
\hline Non-Hispanic white & $78.1(150)$ & $76.6(147)$ & $80.1(161)$ & \\
\hline Household income & & & & .3797 \\
\hline$\geq \$ 40,000$ & $53.5(106)$ & $58.1(115)$ & $55.0(111)$ & \\
\hline$\$ 30,000-\$ 39,000$ & $28.8(57)$ & $21.7(43)$ & $22.3(45)$ & \\
\hline$<\$ 30,000$ & $17.7(35)$ & $20.2(40)$ & $22.8(46)$ & \\
\hline Insurance & & & & .4658 \\
\hline Private & $61.1(121)$ & $62.8(125)$ & $68.5(139)$ & \\
\hline Medicaid & $12.1(24)$ & $14.1(28)$ & $9.8(20)$ & \\
\hline Medicare & $26.8(53)$ & $23.1(46)$ & $21.7(44)$ & \\
\hline Preintervention screening rates & $0(0)$ & $0(0)$ & $0(0)$ & NA \\
\hline
\end{tabular}

Data are \% (n). Small discrepancies between the total number of patients in a group and the summed numbers in the columns reflect data missing for that variable.

*By design, patients without insurance or who were up to date on breast cancer and colorectal cancer screening were excluded from the study.

NA, not applicable.

ration time and postage, was $\$ 7.17$ per patient/ mailing.

For group 2, the cost of automated calls was $\$ 0.09$ per completed call (either a pick-up or an answering machine response). Most patients received 3 waves of calls, and the completion rate (ie, phone picked up or voicemail connected) was $91 \%$. Because each person required multiple automated calls, this intervention cost approximately $\$ 0.25$ per patient, plus $\$ 0.67$ per patient for preparing patient lists, for a total of $\$ 0.92$ per patient.

For group 3, the combined group, the cost was the sum of mailing costs and automated phone calls $(\$ 2.36+\$ 0.92=\$ 3.28 /$ patient for BC screening; $\$ 7.17+\$ 0.92=\$ 8.09 /$ patient for CRC screening).

The cost of automated calls was much less than that of either of the mailings, yet the improvement in screening was similar, suggesting that automated calls were more cost-effective. The cost-effectiveness of the combined intervention compared with the letter only group (ie, the marginal costs divided by the marginal improvement in screening per additional person screened) was $\$ 5.11$ (\$0.92/
0.37-0.19) per additional person screened for BC or $\$ 13.14(0.25 / 0.24-0.17)$ for additional CRC screening.

\section{Discussion}

Our findings from this pragmatic randomized trial are notable in 2 respects. Among insured family medicine patients who were past due for mammography or CRC screening, we observed no differences in screening rates between those who received mailed letters and automated telephone reminders, although each led to absolute increases in screening rates from $13.6 \%$ to $21.6 \%$ among patients who previously were not up to date. Second, the combination of letters and automated telephone reminders yielded improved rates compared with either alone. This suggests that combined outreach to patients past due for cancer screening is superior to either alone.

Few studies have compared letters with automated telephone reminders. Lieu et $\mathrm{al}^{25}$ reported no difference in childhood immunizations between 
Table 3. Unadjusted and Adjusted Postintervention Breast and Colorectal Cancer Screening Rates*

\begin{tabular}{|c|c|c|c|c|}
\hline & \multicolumn{2}{|c|}{ Unadjusted } & \multicolumn{2}{|c|}{ Adjusted $^{\dagger}$} \\
\hline & $\begin{array}{l}\text { Screened } \\
(\%)\end{array}$ & $\begin{array}{c}P \\
\text { Value }^{\ddagger}\end{array}$ & $\begin{array}{c}\text { Screened } \\
\text { (Adjusted \%) }\end{array}$ & $\begin{array}{c}P \\
\text { Value }^{\ddagger}\end{array}$ \\
\hline \multicolumn{5}{|l|}{ Breast cancer screen } \\
\hline Letter & 18.9 & .0062 & 20.3 & .0300 \\
\hline Automated call & 21.6 & .0209 & 24.3 & .0053 \\
\hline $\begin{array}{l}\text { Letter and } \\
\text { automated call }\end{array}$ & 36.6 & & 39.1 & \\
\hline \multicolumn{5}{|l|}{$\begin{array}{l}\text { Colorectal cancer } \\
\text { screen }\end{array}$} \\
\hline Letter & 16.7 & .0519 & 20.0 & .0297 \\
\hline Automated call & 13.6 & .0060 & 16.5 & .0022 \\
\hline $\begin{array}{l}\text { Letter and } \\
\text { automated call }\end{array}$ & 24.1 & & 28.5 & \\
\hline
\end{tabular}

*By definition, preintervention screening rates for each randomization group were $0 \%$ for both mammography and colorectal cancer screening.

${ }^{\dagger}$ Screening rate adjusted for age, race/ethnicity, income, and insurance.

${ }^{\ddagger} P$ values are for the single vs combined intervention.

these strategies. One study of women soon due for mammography reported higher rates following an automated reminder telephone call compared with a mailed reminder letter. ${ }^{26}$ Given the substantial difference in cost, our findings support the use of automated telephone reminders as an alternative to mailed letters if only a single intervention is planned. Practices that are currently mailing reminders to patients past due for screening might consider switching modalities and assess whether they improve screening rates.

The finding that combined modalities yielded improved results is also consistent with some previous studies. ${ }^{25,27-29}$ The addition of automated phone calls to letters nearly doubled rates of mammography screening and increased rates of CRC screening by $8 \%$. Yet in a previous study we found that the use of automated phone calls was of no additional benefit beyond that of a personalized letter alone in improving cancer screening rates $(12.2 \% \text { vs } 15.3 \% ; P>0.05)^{21}$ The difference in findings may have resulted from higher poverty rates in the previous study and correspondingly lower automated phone reminder completion rates ( $82 \%$ vs $91 \%$ ). These differences also could reflect possible differences in patients' responses to automated calls within the 2 practices. Some patients may be more likely to connect the phone call to the letter they received and follow up accordingly, whereas others may ignore telephone messages even if they are received.

Practices that are currently mailing reminders but wish to improve their performance might consider adding automated phone calls. Our finding of a marginal cost for the combined intervention (\$1.57 and \$3.57 per additional patient screened for $\mathrm{BC}$ and CRC, respectively) compare favorably with previous economic assessments of interventions to promote cancer screening. ${ }^{30-34}$

In our combined intervention patients received letters following the automated calls based on the supposition that patients would be "primed" to receive the subsequent letter. We are not aware of empirical data regarding the optimal sequence of reminders for cancer screening. In a study of childhood immunization promotion, however, delivering the automated calls after the letter (rather than before) improved rates. ${ }^{25}$

For mammogram screening, $72.4 \%$ of women (50 to 74 years old) in the general population who completed the National Health Interview Survey in 2010 reported screening in the past 2 years. This is well below the Healthy People 2020 goal of $81.1 \% .{ }^{35}$ In the practice studied here, $67 \%$ of insured women had been screened in the past year. Thus, while for most women the usual education and referral efforts have been effective, new methods are needed to reach the third of women currently not getting screened. A combined approach targeted solely at those not up to date yields improved screening rates compared with either mail or calls alone. The additional cost per patient screened seems modest compared with previous mammography and CRC promotion interventions. ${ }^{30,32,36}$ Nonetheless, the overall outreach response even within the combined group was lower than we had hoped. We previously found relatively greater improvements in cancer screening from combined outreach and point-of-care prompts, suggesting that both outreach and in-reach may be needed to optimize responses. ${ }^{20,21}$ Organized team approaches may also help. ${ }^{37}$

With implementation of the Affordable Care Act, physician revenues are becoming more dependent on achieving certain quality metrics such as cancer screening rates. In the Rochester, New York, area, where the study was conducted, most insurers are tracking mammogram and CRC screening rates. A portion of the supplemental payments to clinicians depends on these quality mea- 
sures. From a practice or individual provider perspective, it is likely that the additional reimbursements expected from meeting these metrics would offset the additional staff time and materials needed for patient outreach.

Limitations of this study include the potential that findings would not be generalizable to other insured groups within primary care practices in the United States. For example, all communication for this study was provided in English. This would not be effective in some practices with substantial nonEnglish-speaking populations, yet providing outreach in other languages also increases the cost of those services. In addition, the cost of identifying insured patients who were overdue for screening was not included in the analysis of cost; however, it is reasonable to assume that electronic tracking of screening by electronic health record systems and payers will increase in the future, making this step automatic for most practices.

\section{Conclusion}

This study shows a significant and additive effect of 2 low-cost interventions to increase cancer screening rates in a primary care practice. These findings are of direct relevance to practices seeking to increase screening rates among patients who remain unscreened with usual care.

\section{References}

1. Centers for Disease Control and Prevention (CDC). Cancer screening - United States, 2010. MMWR Morb Mortal Wkly Rep 2012;61:41-5.

2. Steele CB, Rim SH, Joseph DA, King JB, Seeff LC; Centers for Disease Control and Prevention (CDC). Colorectal cancer incidence and screening - United States, 2008 and 2010. MMWR Surveill Summ 2013;62(Suppl 3):53-60.

3. 2012 National healthcare disparities report. Rockville (MD): Agency for Healthcare Research and Quality; 2013. Available from: http://www.ahrq.gov/research/ findings/nhqrdr/nhdr12/index.html. Accessed November 15, 2014.

4. Breen N, Meissner HI. Toward a system of cancer screening in the United States: trends and opportunities. Annu Rev Public Health 2005;26:561-82.

5. Jepson R, Clegg A, Forbes C, Lewis R, Sowden A, Kleijnen J. The determinants of screening uptake and interventions for increasing uptake: a systematic review. Health Technol Assess 2000;4:i-vii, 1-133.

6. Kho ME, Duffett M, Willison DJ, Cook DJ, Brouwers MC. Written informed consent and selection bias in observational studies using medical records: systematic review. BMJ 2009;338:b866.

7. Junghans C, Jones M. Consent bias in research: how to avoid it. Heart 2007;93:1024-5.

8. Ahmed NU, Haber G, Semenya KA, Hargreaves MK. Randomized controlled trial of mammography intervention in insured very low-income women. Cancer Epidemiol Biomarkers Prev 2010; 19:1790-8.

9. Phillips CE, Rothstein JD, Beaver K, Sherman BJ, Freund KM, Battaglia TA. Patient navigation to increase mammography screening among inner city women. J Gen Intern Med 2011;26:123-9.

10. Jean-Jacques M, Kaleba EO, Gatta JL, Gracia G, Ryan ER, Choucair BN. Program to improve colorectal cancer screening in a low-income, racially diverse population: a randomized controlled trial. Ann Fam Med 2012;10:412-7.

11. Church TR, Yeazel MW, Jones RM, et al. A randomized trial of direct mailing of fecal occult blood tests to increase colorectal cancer screening. J Natl Cancer Inst 2004;96:770-80.

12. Dietrich AJ, Tobin JN, Cassells A, et al. Translation of an efficacious cancer-screening intervention to women enrolled in a Medicaid managed care organization. Ann Fam Med 2007;5:320-7.

13. Lasser KE, Murillo J, Lisboa S, et al. Colorectal cancer screening among ethnically diverse, low-income patients: a randomized controlled trial. Arch Intern Med 2011;171:906-12.

14. Mosen DM, Feldstein AC, Perrin N, et al. Automated telephone calls improved completion of fecal occult blood testing. Med Care 2010;48:604-10.

15. Lantz PM, Stencil D, Lippert MT, Beversdorf S, Jaros L, Remington PL. Breast and cervical cancer screening in a low-income managed care sample: the efficacy of physician letters and phone calls. Am J Public Health 1995;85:834-6.

16. Sequist TD, Zaslavsky AM, Marshall R, Fletcher RH, Ayanian JZ. Patient and physician reminders to promote colorectal cancer screening: a randomized controlled trial. Arch Intern Med 2009;169:364-71.

17. DeFrank JT, Rimer BK, Gierisch JM, Bowling JM, Farrell D, Skinner CS. Impact of mailed and automated telephone reminders on receipt of repeat mammograms: a randomized controlled trial. Am J Prev Med 2009;36:459-67.

18. Simon SR, Zhang F, Soumerai SB, et al. Failure of automated telephone outreach with speech recognition to improve colorectal cancer screening: a randomized controlled trial. Arch Intern Med 2010;170: 264-70.

19. Sabatino SA, Lawrence B, Elder R, et al. Effectiveness of interventions to increase screening for breast, cervical, and colorectal cancers: nine updated systematic reviews for the guide to community preventive services. Am J Prev Med 2012;43:97-118. 
20. Hendren S, Winters P, Humiston S, et al. Randomized, controlled trial of a multimodal intervention to improve cancer screening rates in a safety-net primary care practice. J Gen Intern Med 2013;29:41-9.

21. Fortuna RJ, Idris A, Winters P, et al. Get screened: a randomized trial of the incremental benefits of reminders, recall, and outreach on cancer screening. J Gen Intern Med 2014;29:90-7.

22. US Preventive Services Task Force. Screening for breast cancer: U.S. Preventive Services Task Force recommendation statement. Ann Intern Med 2009; 151:716-26.

23. Rauscher GH, Johnson TP, Cho YI, Walk JA. Accuracy of self-reported cancer-screening histories: a meta-analysis. Cancer Epidemiol Biomarkers Prev 2008;17:748-57.

24. Fiscella K, Holt K, Meldrum S, Franks P. Disparities in preventive procedures: comparisons of self-report and Medicare claims data. BMC Health Serv Res 2006;6:122.

25. Lieu TA, Capra AM, Makol J, Black SB, Shinefield HR. Effectiveness and cost-effectiveness of letters, automated telephone messages, or both for underimmunized children in a health maintenance organization. Pediatrics 1998;101:E3.

26. DeFrank JT, Rimer BK, Gierisch JM, Bowling JM, Farrell D, Skinner CS. Impact of mailed and automated telephone reminders on receipt of repeat mammograms: a randomized controlled trial. Am J Prev Med 2009;36(6):459-67.

27. Gardner MP, Adams A, Jeffreys M. Interventions to increase the uptake of mammography amongst low income women: a systematic review and meta-analysis. PLoS One 2013;8:e55574.

28. Green BB, Wang CY, Anderson ML, et al. An automated intervention with stepped increases in support to increase uptake of colorectal cancer screen- ing: a randomized trial. Ann Intern Med 2013;158(5 Pt 1):301-11.

29. Feldstein AC, Perrin N, Rosales AG, et al. Effect of a multimodal reminder program on repeat mammogram screening. Am J Prev Med 2009;37:94-101.

30. Fishman P, Taplin S, Meyer D, Barlow W. Costeffectiveness of strategies to enhance mammography use. Eff Clin Pract 2000;3:213-20.

31. Smith DH, Feldstein AC, Perrin N, et al. Automated telephone calls to enhance colorectal cancer screening: economic analysis. Am J Manag Care 2012;18: 691-9.

32. Sequist TD, Franz C, Ayanian JZ. Cost-effectiveness of patient mailings to promote colorectal cancer screening. Med Care 2010;48:553-7.

33. Lewis CL, Brenner AT, Griffith JM, Moore CG, Pignone MP. Two controlled trials to determine the effectiveness of a mailed intervention to increase colon cancer screening. N C Med J 2012;73:93-8.

34. Misra S, Lairson DR, Chan W, et al. Cost effectiveness of interventions to promote screening for colorectal cancer: a randomized trial. J Prev Med Public Health 2011;44:101-10.

35. HealthyPeople.gov. 2020 topics \& objectives: cancer. November 14, 2014. Available from: http:// www.healthypeople.gov/2020/topicsobjectives2020/ objectiveslist.aspx? topicId $=5$. Accessed November 15, 2014.

36. Saywell RM Jr, Champion VL, Skinner CS, McQuillen D, Martin D, Maraj M. Cost-effectiveness comparison of five interventions to increase mammography screening. Prev Med 1999;29:37482.

37. Klabunde CN, Lanier D, Breslau ES, et al. Improving colorectal cancer screening in primary care practice: innovative strategies and future directions. J Gen Intern Med 2007;22:1195-205. 